\title{
Crystal structure of erythro-2-hydroxy-3-phenylbutyric acid, $\mathrm{C}_{6} \mathrm{H}_{5} \mathrm{CH}\left(\mathrm{CH}_{3}\right) \mathrm{CH}(\mathrm{OH}) \mathrm{COOH}$
}

\author{
R. Ludwig* and D. Lentz* \\ Freie Universität Berlin. Institut für Chemie / Analytische und Anorganische Chemie, Fabeckstr. 34-36, D-14195 Berlin, Germany
}

Received September 18, 2001, accepted January 24, 2002; CCDC-No. 1267/735

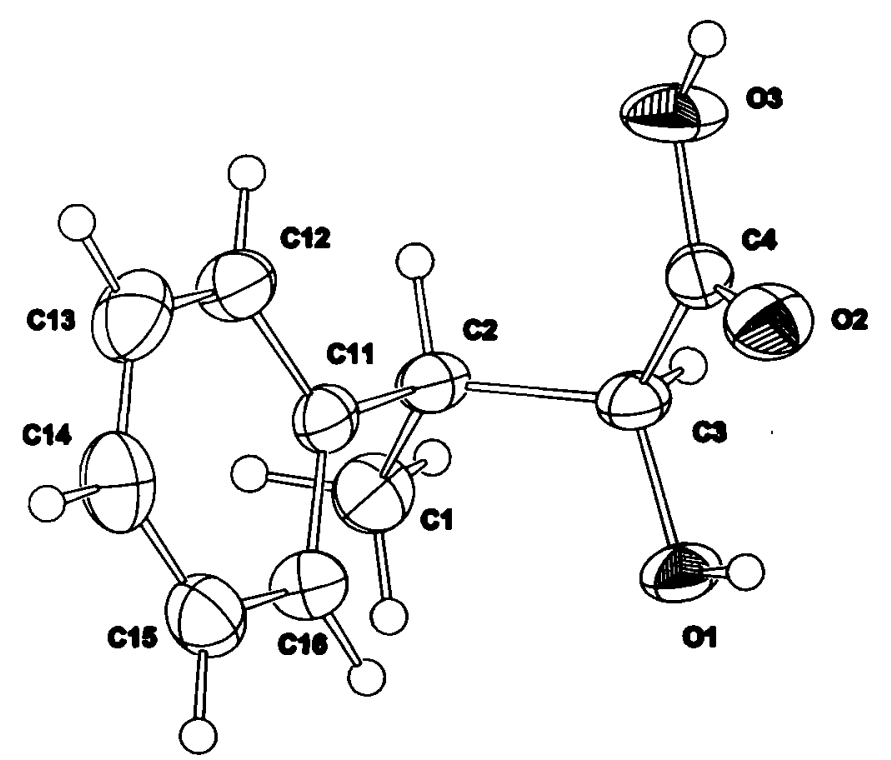

Abstract

$\mathrm{C}_{10} \mathrm{H}_{12} \mathrm{O}_{3}$, monoclinic, $P 12_{1} / c \mathrm{l}$ (No. 14), $a=8.427(2) \AA$, $b=5.967(2) \AA, c=18.008(2) \AA, \beta=90.43(2)^{\circ}, V=905.5 \AA^{3}$, $Z=4, R_{\mathrm{gl}}(F)=0.033, w R_{\mathrm{all}}\left(F^{2}\right)=0.096, T=293 \mathrm{~K}$.

\section{Source of material}

The title compound was synthesized from the hydrogensulfite adduct of 2-phenylpropionaldehyde (Merck) via the corresponding nitrile [1]. It was crystallized 8 times from toluene to separate it from the more soluble threo-form, and twice from water (mp $395 \mathrm{~K}$, Lit. $395 \mathrm{~K}$ for the racemate). NMR spectroscopic data is included in the deposited CIF-file.

\section{Discussion}

$\alpha$-Hydroxycarboxylic acids are of interest as complexing ligands, e.g. for lanthanoids [2]. In these studies, it turned out that the stability of adducts between a lanthanoid ion, a $\beta$-diketone and an $\alpha$-hydroxycarboxylic acid is influenced by the structure of the latter. The adduct formation can improve the mutual separation and applied for analytical purposes. We were able to purify one of the diatereomers of 2-hydroxy-3-phenylbutyric acid [3] by fractionated crystallization. The erythro-isomer is less soluble in toluene compared with the threo form.

Table 1. Data collection and handling.

\begin{tabular}{ll}
\hline Crystal: & colourless needle, \\
& size $0.2 \times 0.3 \times 0.8 \mathrm{~mm}$ \\
Wavelength: & Mo $K_{a}$ radiation $(0.71069 \AA)$ \\
$\mu:$ & $0.97 \mathrm{~cm}^{-1}$ \\
Diffractometer, scan mode: & Stoe, $\omega$ \\
$2 \theta_{\max }:$ & $52^{\circ}$ \\
$N(h k l)_{\text {measured }}, N(h k l)_{\text {unique: }}:$ & 1916,1790 \\
Criterion for $J_{\text {obs, }} N(h k l)_{\text {gt: }}:$ & $I_{\text {obs }}>2 \sigma\left(I_{\text {obs }}\right), 1172$ \\
$N(\text { param })_{\text {hefined: }}$ & 167 \\
Programs: & SHELXS-86 [4], SHELXL-93 [5]
\end{tabular}

Table 2. Atomic coordinates and displacement parameters (in $\AA^{2}$ ).

\begin{tabular}{llllll}
\hline Atom & Site & $x$ & $y$ & $z$ & $U_{\text {iso }}$ \\
\hline H(1A) & $4 e$ & $0.654(3)$ & $0.066(4)$ & $0.472(1)$ & $0.058(6)$ \\
H(1B) & $4 e$ & $0.745(2)$ & $-0.079(4)$ & $0.532(1)$ & $0.048(5)$ \\
H(1C) & $4 e$ & $0.837(2)$ & $0.063(3)$ & $0.469(1)$ & $0.049(5)$ \\
H(2) & $4 e$ & $0.756(2)$ & $0.399(3)$ & $0.5249(9)$ & $0.031(4)$ \\
H(3) & $4 e$ & $0.995(2)$ & $0.269(3)$ & $0.5641(9)$ & $0.028(4)$ \\
H(12) & $4 e$ & $0.544(2)$ & $0.591(4)$ & $0.570(1)$ & $0.046(5)$ \\
H(13) & $4 e$ & $0.315(3)$ & $0.622(4)$ & $0.640(1)$ & $0.060(6)$ \\
H(14) & $4 e$ & $0.232(2)$ & $0.325(3)$ & $0.715(1)$ & $0.046(5)$ \\
H(15) & $4 e$ & $0.391(2)$ & $-0.007(4)$ & $0.720(1)$ & $0.053(6)$ \\
H(16) & $4 e$ & $0.618(2)$ & $-0.026(3)$ & $0.650(1)$ & $0.044(5)$ \\
H(10) & $4 e$ & $0.947(3)$ & $0.123(5)$ & $0.691(2)$ & $0.089(9)$ \\
H(30) & $4 e$ & $0.936(3)$ & $0.789(5)$ & $0.628(1)$ & $0.073(7)$ \\
\hline
\end{tabular}

\footnotetext{
* (e-mails: rludwig@mail.chemie.fu-berlin.de, lentz@chemie.fu-berlin.de)
} 
Table 3. Atomic coordinates and displacement parameters (in $\AA^{2}$ ).

\begin{tabular}{|c|c|c|c|c|c|c|c|c|c|c|}
\hline Atom & Site & $x$ & $y$ & $z$ & $U_{11}$ & $U_{22}$ & $U_{33}$ & $U_{12}$ & $U_{13}$ & $U_{23}$ \\
\hline$O(1)$ & $4 e$ & $0.9226(2)$ & $0.0811(2)$ & $0.64847(7)$ & $0.0504(8)$ & $0.0176(6)$ & $0.0420(8)$ & $0.0027(5)$ & $-0.0149(6)$ & $-0.0001(5)$ \\
\hline$O(2)$ & $4 e$ & $0.9354(2)$ & $0.4880(2)$ & $0.71146(7)$ & $0.0568(8)$ & $0.0297(7)$ & $0.0411(8)$ & $0.0005(6)$ & $-0.0086(6)$ & $-0.0075(5)$ \\
\hline$O(3)$ & $4 e$ & $0.9320(2)$ & $0.6593(2)$ & $0.60114(7)$ & $0.078(1)$ & $0.0179(6)$ & $0.0493(8)$ & $-0.0024(6)$ & $-0.0055(7)$ & $0.0014(6)$ \\
\hline$C(1)$ & $4 e$ & $0.7483(2)$ & $0.0607(3)$ & $0.5037(1)$ & $0.047(1)$ & $0.034(1)$ & $0.035(1)$ & $0.0017(8)$ & $-0.0058(9)$ & $-0.0081(8)$ \\
\hline$C(2)$ & $4 e$ & $0.7530(2)$ & $0.2648(3)$ & $0.55542(9)$ & $0.0363(9)$ & $0.0227(8)$ & $0.0278(8)$ & $0.0016(7)$ & $-0.0026(7)$ & $0.0019(7)$ \\
\hline$C(3)$ & $4 e$ & $0.9097(2)$ & $0.2687(3)$ & $0.60068(9)$ & $0.0337(9)$ & $0.0191(7)$ & $0.0332(9)$ & $0.0028(7)$ & $-0.0005(7)$ & $0.0001(7)$ \\
\hline$C(11)$ & $4 e$ & $0.6059(2)$ & $0.2812(3)$ & $0.60293(8)$ & $0.0325(8)$ & $0.0275(8)$ & $0.0285(8)$ & $0.0001(7)$ & $-0.0075(6)$ & $-0.0026(7)$ \\
\hline$C(12)$ & $4 e$ & $0.5120(2)$ & $0.4721(3)$ & $0.6002(1)$ & $0.041(1)$ & $0.0312(9)$ & $0.042(1)$ & $0.0035(8)$ & $-0.0046(8)$ & $0.0025(8)$ \\
\hline$C(13)$ & $4 e$ & $0.3740(2)$ & $0.4881(4)$ & $0.6407(1)$ & $0.039(1)$ & $0.047(1)$ & $0.056(1)$ & $0.0138(9)$ & $-0.0035(9)$ & $-0.005(1)$ \\
\hline$C(14)$ & 4e & $0.3276(2)$ & $0.3126(4)$ & $0.6856(1)$ & $0.0319(9)$ & $0.062(1)$ & $0.044(1)$ & $-0.002(1)$ & $0.0019(8)$ & $-0.006(1)$ \\
\hline$C(15)$ & $4 e$ & $0.4193(2)$ & $0.1230(4)$ & $0.6895(1)$ & $0.041(1)$ & $0.048(1)$ & $0.041(1)$ & $-0.0081(9)$ & $-0.0012(8)$ & $0.0050(9)$ \\
\hline$C(16)$ & $4 e$ & $0.5566(2)$ & $0.1064(3)$ & $0.6486(1)$ & $0.039(1)$ & $0.0303(9)$ & $0.042(1)$ & $0.0008(8)$ & $-0.0034(8)$ & $0.0035(8)$ \\
\hline
\end{tabular}

Acknowledgment. A grant by Deutsche Forschungsgemeinschaft for R. L. is highly appreciated.

\section{References}

1. Corson, B. B.; Dodge, R. A.; Harris, S. A.; Yeaw, J. S.: Mandelic acid. Org. Synth. Coll. I (1956) 336-340.

2. Hasegawa, Y.; Harada, Y.; Ludwig, R.:Application of $\alpha$-hydroxycarboxylic acids as masking and synergistic agents in mutual separation of lanthanoids(III). Int. Symp. Trace Analysis; Tokyo Univ., 31.7.-1.8. Japan 1998.
3. Beilstein Handbook of organic chemistry. Springer Verlag, Berlin 1971, 4. Ed. Vol. 10, Suppl. E III, 595.

4. Sheldrick, G. M.: Phase Annealing in SHELX-90: Direct methods for Larger Structure. Acta Crystallogr. A46 (1990) 467-473.

5. Sheldrick, G. M.: SHELXL-93, a program for refining crystal structures. University of Göttingen, pre-release version, Germany 1992 